\title{
The influence of peripheral stimuli on the amount and direction of autokinesis
}

\author{
E. J. MACDUFFEE, C. L. SHUPERT, and H. W. LEIBOWITZ \\ The Pennsylvania State University, University Park, Pennsylvania
}

\begin{abstract}
Peripheral visual stimuli were presented in conjunction with an autokinetic target to determine the influence of additional visual contour on the amount and direction of autokinetic movement. The addition of any peripheral stimulus resulted in a significant decrease in autokinesis. The greatest reduction occurred when peripheral stimuli were presented at near eccentricities and when the peripheral stimulus completely surrounded the autokinetic stimulus. Peripheral stimuli also influenced the direction of autokinesis. Stimuli placed at $1^{\circ}$ eccentric to the autokinetic stimulus increased the amount of autokinetic motion in the direction away from the additional stimulus. Also, at more peripheral placements, a vertical bar increased autokinetic movement in the vertical plane. These results are interpreted within the context of the contribution of additional stimuli in the visual field to enhanced fixational stability.
\end{abstract}

Autokinesis (AK) is the apparent motion exhibited by small visual targets on homogeneous backgrounds. A number of different types of variables, including the size and brightness of the autokinetic stimulus (AKS) itself, have been reported to influence the amount of AK (see Levy, 1972, and Royce, Carran, Aftanas, Lehman, \& Blumenthal, 1966, for extensive reviews). It is also well known that the presence of other visual contours in addition to the AKS reduces the amount of AK (Edwards, 1954; Graybiel \& Clark, 1945; Karwoski, Redner, \& Wood, 1948; Royce, Stayton, \& Kinkade, 1962). In these studies, additional stimuli, in the form of a lighted cross (Karwoski et al., 1948) or a matrix or circle of lighted points (Edwards, 1954; Royce et al., 1962), were placed $2^{\circ}$ to $20^{\circ}$ eccentric to the AKS, which was a lighted point in all cases. Despite differences in procedure, the findings of these studies were quite consistent. Placing additional stimuli in the visual field increased latency to the onset of AK and decreased its perceived extent.

Post, Leibowitz, and Shupert (1982) confirmed and extended these findings. Previous studies had relied on such dependent measures as estimates of the distance traveled by the AKS (Edwards, 1954) and hand-drawn reproductions of the path of the apparent movement (Karwoski et al., 1948). Such measures are difficult to quantify and are easily influenced by suggestion (see Leibowitz, Shupert, Post, \& Dichgans, 1983, and Levy, 1972, for discussions). Also, the stimulus displays in the previous experiments had been limited to less than $20^{\circ}$ of arc. In order to determine the effect of additional stimuli placed further in the periphery, Post et al. (1982) placed a second point of light adjacent to an AKS at eccentricities that varied from $1^{\circ}$ to $60^{\circ}$ in the horizontal plane. Observers

This work was supported by National Eye Institute Grant 03276 . Reprint requests should be directed to H. W. Leibowitz, Psychology Department, The Pennsylvania State University, University Park, PA 16802. E. J. MacDuffee can be reached c/o H. W. Leibowitz. used the sound of a metronome ticking at $1 \mathrm{~Hz}$ to estimate the duration of $\mathrm{AK}$ in each trial. The duration of AK decreased as the distance between the AKS and the peripheral stimulus decreased, with significantly less than baseline AK observed when the peripheral light was placed at $1^{\circ}$ eccentric to the AKS.

Post et al. (1982) did not specifically examine the effect of additional stimuli on the direction of AK. However, the findings of other studies indicate that the direction of AK may also be influenced by peripheral stimuli. Uchiyama and Tsuji (1963) briefly presented a small $\left(1^{\circ}\right)$ geometric figure $7^{\prime}$ to $34^{\prime}$ of arc away from an AKS. Observers reported that the AKS appeared to move swiftly away from the figure; this effect was more pronounced for closer placements of the peripheral figure. Sadler, Mefferd, and Weiland (1966) presented observers with an AKS in the center of two concentric circles of eight lights (subtending $43^{\prime}$ and $1^{\circ} 26^{\prime}$ of arc). On the basis of drawings the observers made of the path of the apparent motion, Sadler et al. reported that the proportion of movement in the plane of any peripheral light increased significantly from baseline whenever an additional light was present.

Although a variety of hypotheses have been proposed to account for $\mathrm{AK}$, the most widely accepted theories attribute $\mathrm{AK}$ to a loss of fixational stability in the impoverished visual environments in which AK is typically observed. AK is thought to result either from a misinterpretation, or a subtle increase, in the amount of retinal image motion resulting from the small eye movements that occur constantly during attempted fixation (Matin \& MacKinnon, 1964), or from an increased need to rely on voluntary, rather than reflexive, fixational mechanisms (Leibowitz et al., 1983; Post \& Leibowitz, 1985). Thus, peripheral stimuli that decrease the amount of AK are thought to enhance fixational stability generally. Those that result in more movement in a particular direction are 
thought to provide less information about fixational stability in that direction, or to destabilize fixation selectively (see Leibowitz et al., 1983, and Post et al., 1982, for discussions).

The purpose of the present study was to determine whether increases in the size of additional stimuli in the visual field would result in greater reductions in AK, or significant reductions in AK at more peripheral placements in the visual field, than those found by Post et al. (1982). The shape of the peripheral stimulus was also varied to determine whether shapes that provide more effective feedback about fixational stability in particular directions reduce the amount of AK in those directions. Specifically, we compared the effects of squares, vertically oriented bars, and horizontally oriented bars of equal area on the AK of a small point of light. The results are interpreted in terms of the contribution of peripheral stimuli to increased fixational stability in impoverished visual environments.

\section{EXPERIMENT 1}

\section{Method}

Observers. Eight female volunteers, aged 17 to 18 years, participated in the study. All had a minimum corrected visual acuity of 20/20, with no abnormal phorias as determined by a Titmus vision tester. They observed AK for approximately 2 min of practice time prior to participating, but were naive as to the purpose of the experiment.

Procedure. There were three sessions separated by at least $24 \mathrm{~h}$. Observers were tested individually. All were right-eye dominant as determined by a sighting task, and observations were made monocularly with the dominant eye. The observer was seated with her head in a chinrest facing a display consisting of a metal strip curved in a semicircular arc and mounted on a wooden stand so that a distance of $50 \mathrm{~cm}$ was maintained from any point on the strip to the observer's right eye. The AKS, which was produced by masking an electroluminescent panel to obtain a small point of light subtending $14^{\prime}$ of arc, was maintained at a fixed position on the display directly in front of the observer's right eye.

Peripheral stimuli were produced by masking electroluminescent panels to obtain three sizes of three shapes: a square, a horizontal bar, and a vertical bar. The bar stimuli subtended $17^{\prime} \times 1^{\circ} 9^{\prime}$, $34^{\prime} \times 9^{\circ}$, and $34^{\prime} \times 32^{\circ} 37^{\prime}$ of arc. The sides of the square stimuli subtended $34^{\prime}, 2^{\circ} 17^{\prime}$, and $4^{\circ} 34^{\prime}$ of arc. In each session, all three shapes of one of the three sizes were presented at four peripheral eccentricities: $1^{\circ}, 10^{\circ}, 30^{\circ}$, and $60^{\circ}$. Thus, of the 36 possible combinations of nine peripheral stimuli placed at four eccentricities, only 12 were presented in a given session, and each of these 12 combinations was presented for three 20 -sec trials. All of the stimuli had a luminance of $2.66 \mathrm{~cd} / \mathrm{m}^{2}$, and the peripheral stimuli were flashed at a rate of $1.5 \mathrm{sec}$ on and $.5 \mathrm{sec}$ off to prevent Troxler fading.

During all trials observers used the sound of a metronome ticking at $1 \mathrm{~Hz}$ to count the number of seconds during which the AKS appeared to move. Directions of movement were reported in terms of the eight major compass points (north, south, east, west, and the diagonals). Following each 20 -sec trial, the room lights were turned on and the volunteers reported the number of seconds AK was observed in each direction. Observers practiced this procedure for approximately 2 min prior to participating in the experiment and reported no difficulty with the task. The order of presentation of the three shapes at the four eccentricities and the baseline trials in each session were approximately counterbalanced.

\section{Results and Discussion}

The total time AK was observed in each of eight directions in a 60-sec period (three 20-sec trials for each stimulus variation in each session) was computed for each observer. The data were analyzed using a four-way analysis of variance (shape $\times$ size $\times$ eccentricity $\times$ direction). All subsequent comparisons were made using Fisher's least significant difference $(L S D)$ procedure.

Within the range of sizes tested here, the small stimuli were as effective as the large stimuli at reducing $\mathrm{AK}$ $[F(2,14)=.09, p=.9134]$. Therefore, all subsequent results have been collapsed across size. Figure 1 presents the mean total movement (out of $60 \mathrm{sec}$ ) for all peripheral stimulus configurations and for all observers as a function of the eccentricity of the second stimulus. The greatest reduction in $\mathrm{AK}$ occurred when a second stimulus was placed at $1^{\circ}$, whereas stimuli placed at more peripheral eccentricities were less effective in reducing $\operatorname{AK}[F(3,21)$ $=18.47, p<.001]$. These results are consistent with those reported by Post et al. (1982), and can be attributed to an increase in fixational stability. In contrast to the findings of Post et al., however, the total amount of movement of the AKS observed for each position of the peripheral stimulus was significantly reduced from baseline $(p<.05)$ and was also significantly different $(p<.05)$ from that observed at every other eccentricity, except that the amounts of movement at $30^{\circ}$ and $60^{\circ}$ did not significantly differ from each other. Thus, while the maximum reduction of $\mathrm{AK}$ occurred when the peripheral stimulus was placed at near eccentricities, significant reduction in AK was also demonstrated when the second stimulus was placed more peripherally in the visual field. Since the smallest stimulus in the present study was larger than the single additional light point used by Post et al., these results are consistent with the hypothesis that larger amounts of visual contour provide a greater degree of fixational stability than do small amounts of contour.

In all stimulus conditions, movement to the north was found to predominate $[F(7,49)=6.06, p<.001]$, with relatively less movement in the horizontal and diagonal directions. This effect is typical and has been well documented (see Levy, 1972). However, the shape of the peripheral stimulus selectively affected the direction of $\operatorname{AK}[F(14,98)=4.08, p<.001]$. As Table 1 shows, significantly more north and south movement was observed when the peripheral stimulus was a vertical bar than when it was a horizontal bar $(p<.05)$. Also, significantly more northward movement $(p<.05)$ was observed when the stimulus was a vertical bar than when it was a square. This finding is consistent with the hypothesis that the vertical stimulus would be most effective at increasing fixational stability in the horizontal plane of the visual field. Thus, the dimension in which gaze was least stable (the vertical one) was the one in which the most AK was observed. This result is consistent with the findings of Sadler et al. (1966), who reported an increase in $\mathrm{AK}$ in the plane of any peripheral light. On the basis of these findings, it would be predicted that $\mathrm{AK}$ in the 


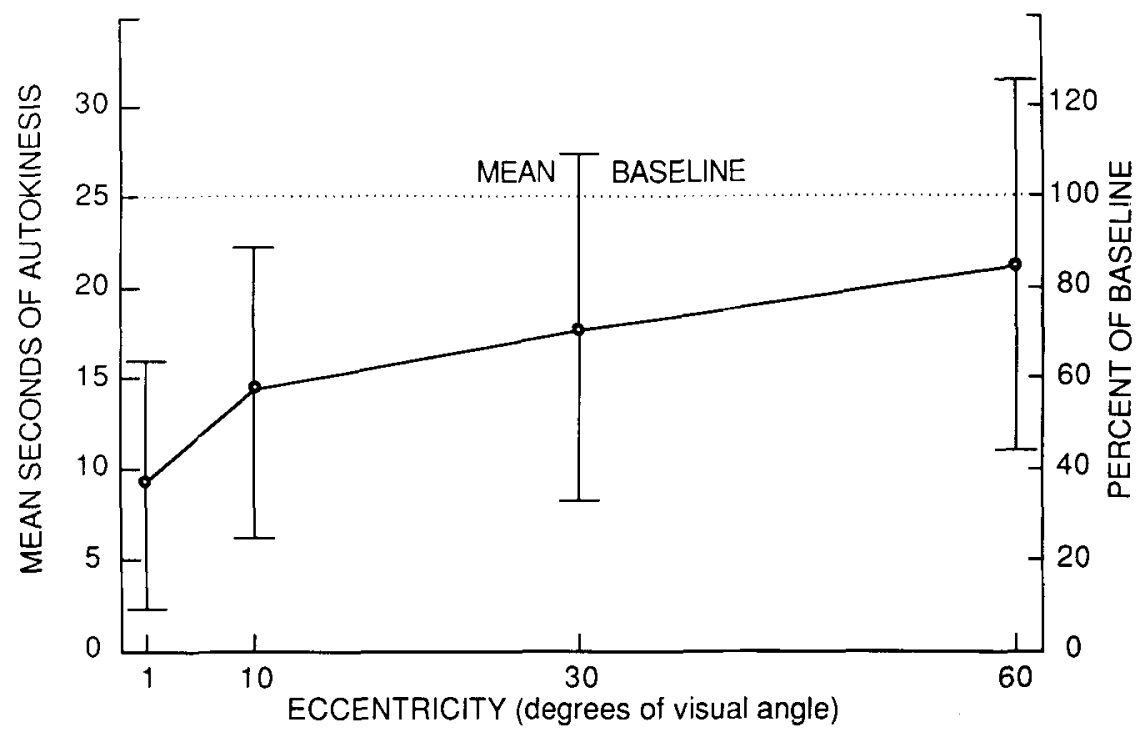

Figure 1. Mean seconds of autokinesis reported in a 60-sec observation period and percent of baseline, as a function of the eccentricity of the peripherally placed additional stimuli, with standard error bars indicated.

horizontal dimension would be increased when the peripheral stimulus was a horizontal bar. Our data provide partial support for this prediction: The most eastward movement was observed when the peripheral stimulus was a horizontal bar; however, this difference was directional but not statistically significant $(p<.1)$.

It is possible that the stimulus configuration used in this experiment led to the failure to find increased horizontal AK in the presence of the horizontal stimulus. The AKS was positioned near the midpoint of the bar when the peripheral stimulus was a vertical bar, but at one end of the bar stimulus when it was horizontal. If the horizontal bar had been placed below or above the AKS at about the midpoint, it would have more closely approximated the configuration of the vertical bar and the AKS. This alternative arrangement of the vertical bar and the AKS might have been a better stimulus for fixational stability in the north and south directions, because it would have

Table 1

Mean Seconds of Autokinesis Reported in Eight Directions as a Function of the Shape of the Peripheral Stimulus

\begin{tabular}{lccc}
\hline & \multicolumn{3}{c}{ Peripheral Stimulus Shape } \\
\cline { 2 - 2 } Direction & Vertical & Horizontal & Square \\
\hline North & 8.60 & 4.71 & 4.76 \\
South & 3.99 & 2.30 & 3.14 \\
East & 2.24 & 3.67 & 2.74 \\
West & 1.36 & 1.49 & 1.50 \\
Northeast & .66 & 1.34 & .98 \\
Northwest & .55 & .67 & .32 \\
Southeast & .21 & .32 & .09 \\
Southwest & .31 & .65 & .25 \\
\hline
\end{tabular}

Note-Critical value for Fisher's $L S D$ comparisons $=1.69$. Differences that exceed this value are significant at the .05 level. provided a continuous fixational referent for gaze stability in this dimension of the visual field. Westward movement was observed for approximately equal amounts of time for all three stimulus shapes. There was little movement in the diagonal directions, and shape did not significantly affect these directions.

The direction of AK was also influenced by the eccentricity of the peripheral stimulus. Figure 2 shows that movement to the north, south, east, and northeast all increased with greater eccentricity of the peripheral stimulus. The total amount of westward movement, however, decreased as the eccentricity of the peripheral stimulus increased $[F(21,147)=2.75, p<.001]$. There was little movement in the other diagonal directions, and the amount of movement did not change systematically as a function of the eccentricity of the peripheral stimulus. The fact that the most westward movement was observed when the peripheral stimulus was placed at $1^{\circ}$ eccentricity (see Figure 2) is consistent with the findings of Uchiyama and Tsuji (1963), who reported that an AKS appeared to move swiftly away from the side of another figure when both stimuli were presented foveally. Since all stimuli in the present study were placed to the east of the AKS, only westward movement represented $\mathrm{AK}$ in the direction away from the peripheral stimulus. Although Uchiyama and Tsuji attributed this type of AK motion to the "field force" of the peripherally placed figures, these results may also depend on the influence of peripheral stimuli on fixational stability. Stimuli presented very close to the AKS may increase the tendency of the eyes to drift in their direction. In this case, it would be predicted that $\mathrm{AK}$ would be observed predominantly in the direction away from the peripheral stimulus. 


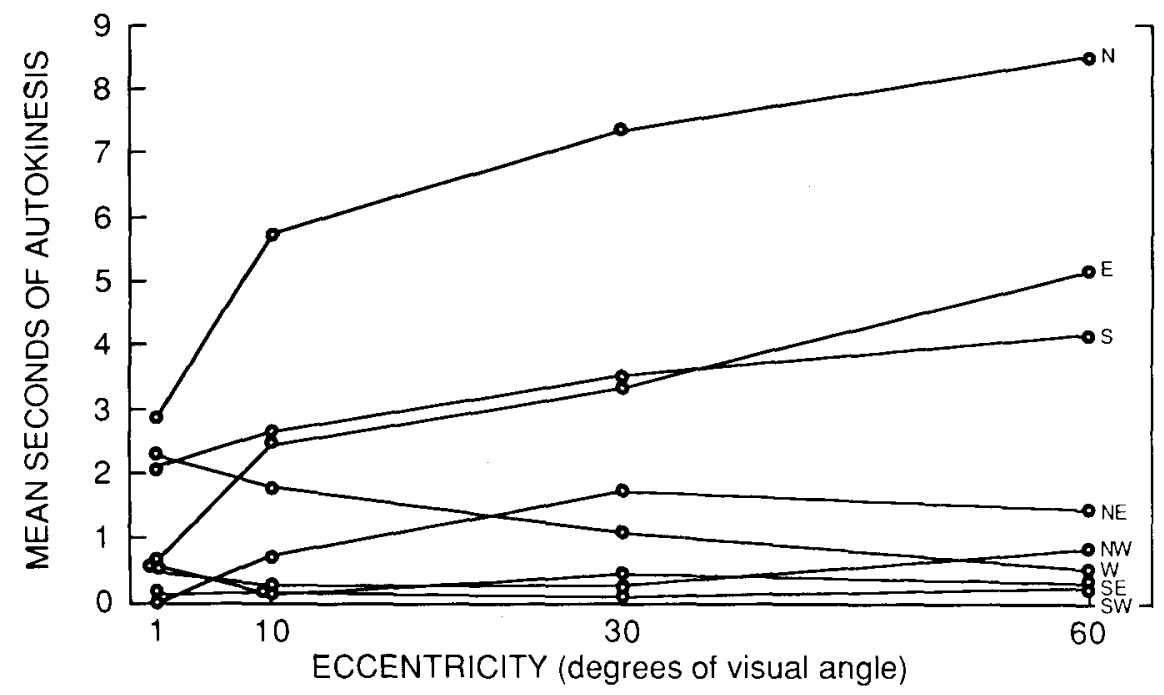

Figure 2. Mean seconds of autokinesis reported in a 60-sec observation period in each of eight directions, as a function of the eccentricity of the peripherally placed additional stimuli.

\section{EXPERIMENT 2}

Experiment 1 demonstrated that the greatest reduction in AK occurred when peripheral stimuli were presented in the near periphery. However, even stimuli placed $1^{\circ}$ eccentric to the AKS produced only a $60 \%$ reduction in AK. The purpose of Experiment 2 was to determine the characteristics of peripheral stimuli that would further reduce $\mathrm{AK}$. We therefore compared the effects of peripheral stimuli that were all presented $5^{\circ}$ eccentric to the AKS but were varied in shape so as to place different amounts of contour in the near periphery. Both a square and a circular annulus, providing identical amounts of contour, were employed to determine whether a square, which provides absolute horizontal and vertical directional referents, would be more effective in reducing AK than a circle, which provides more ambiguous directional cues.

In Experiment 1 there was a larger proportion of movement in the direction away from the additional stimulus when that stimulus was placed in the near periphery. Therefore, in Experiment 2, the bar and the arc were placed above, below, to the left of, and to the right of the AKS, in order to determine whether the AKS would appear to move away from the peripheral stimulus in each of these stimulus configurations.

\section{Method}

Observers. Five male and 5 female volunteers who had not participated in Experiment 1 were the observers. They were between the ages of 17 and 18 and each had a minimum corrected visual acuity of 20/20, with no abnormal phorias. As in the previous study, they observed AK for approximately 2 min before participating, but were naive as to the purpose of the experiment.

Procedure. All observers were right-eye dominant as determined by a sighting task, and observations were made monocularly with the dominant eye. The particulars of the display and the positioning of the observers were as in Experiment 1. Both the stimuli and the dependent measure, however, were different. The peripheral stimuli in this study were varied both in shape and position. The shapes were a square annulus, a circular annulus, a bar curved in the shape of a semicircular arc, and a straight bar (widths were 17' of arc). These stimuli were produced by masking electroluminescent panels, as in Experiment 1. The AKS was a small dot of phosphorescent paint on a black background (subtending 14' of arc). The AKS was placed within the annular stimuli at a distance of $5^{\circ}$ from the inner edges. The bar and the arc were placed in one of four positions $-5^{\circ}$ above, below, to the left, or to the right of the AKS. The square and circular annuli subtended $9^{\circ} \times 9^{\circ}$ of arc, the semicircular $\operatorname{arc} 4^{\circ} 34^{\prime} \times 9^{\circ}$, and the bar $17^{\prime} \times 9^{\circ}$. Autokinetic and peripheral stimuli had a luminance of $.34 \mathrm{~cd} / \mathrm{m}^{2}$.

The experiment lasted $1 \mathrm{~h}$ and consisted of four 20-sec trials for each of the 10 conditions described above and eight 20 -sec trials for the AKS in the absence of any other stimuli (baseline condition). A metronome was ticking at $1 \mathrm{~Hz}$ during all trials, and the observers were asked to report the directions of movement in terms of the 12 clock positions. The observers practiced this method for approximately 2 min prior to participating in the experiment and reported no difficulties with the task. The order of all conditions was approximately counterbalanced.

\section{Results and Discussion}

The total time for which AK was reported in an 80-sec period (four 20-sec trials) was computed for each observer. Because of the small amount of diagonal AK reported in Experiment 1, the reported directions of $A K$ in Experiment 2 were combined in the following manner: Reports of movement toward 11:00, 12:00, and 1:00 were combined and designated as northward movement; toward 2:00, 3:00, and 4:00, eastward; toward 5:00, 6:00, and 7:00, southward; and toward 8:00, 9:00, and 10:00, westward. For the conditions in which either the bar or the arc was presented, the position of the peripheral stimulus varied. Consequently, these data were analyzed using a three-way analysis of variance (shape $x$ position $x$ direction). In the conditions in which a square or cir- 


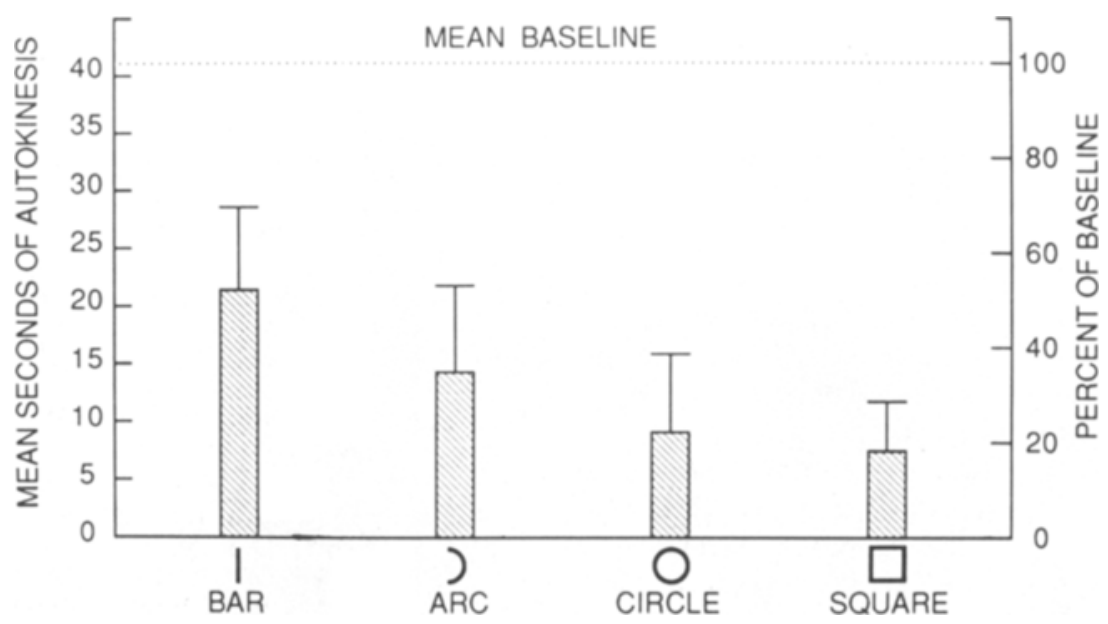

Figure 3. Mean seconds of autokinesis reported in an 80 -sec observation period and percent of baseline, as a function of the shape of the peripherally placed additional stimuli, with standard error bars indicated.

cular annulus was presented, there was only one position for the peripheral stimulus, so a separate two-way anal$y$ sis of variance (shape $x$ direction) was performed on the data for all four shapes combined.

Figure 3 presents the mean number of seconds of $A K$ reported by all observers as a function of the shape of the additional stimuli. The analysis of variance revealed that the effect of shape on the amount of AK was significant $[F(3,27)=9.44, p<.001]$. The annuli, which provided the most additional contour in the foveal and perifoveal regions, reduced AK most effectively (by about $80 \%)$, and did so about equally well $(p<.05)$. The arc produced a reduction of movement that fell along a continuum between that produced by the annuli and that produced by the bar, but was not significantly different from either. The bar produced significantly $(p<.05)$ less reduction in AK than did either annulus, but did not differ significantly from the arc. All shapes significantly $(p<.05)$ reduced AK from baseline. These results are consistent with the hypothesis that additional contour presented near to the AKS enhances fixational stability and therefore results in a reduction of $\mathrm{AK}$. The square and the circular annuli did not differ in their effects on either the amount or the direction of AK. This indicates that the total amount of contour placed near to the AKS is a more salient factor for the systems that stabilize gaze than the type (curved or straight edges) of contour that is presented.

The position of the bar and the arc (above, below, left, or right) did have a sigificant effect on the direction of AK $[F(9,81)=2.06, p<.05]$. The significant interaction was due to the fact that there was more $\mathrm{AK}$ in the southward direction when the peripheral stimuli were placed above the AKS than when the directions of AK and the corresponding positions of the peripheral stimulus were, respectively, west and left, north and left, east and right, west and above, and west and right. None of these significant differences support the hypothesis that stimuli placed near the AKS increase the amount of AK in the direction away from the peripheral stimulus. For instance, southward movement when stimuli were placed above the AKS did not differ significantly from southward movement when stimuli were placed below the AKS, as would have been predicted.

\section{GENERAL DISCUSSION}

The results of the present experiments both confirm and extend the previous findings that additional contour in the visual field can influence the amount of AK reported (Edwards, 1954; Karwoski et al., 1948; Post et al., 1982; Royce et al., 1962). The results of Experiments 1 and 2, taken together, show that increasing the amount of additional contour in the central visual field can significantly reduce the amount of $\mathrm{AK}$ reported. In Experiment 1, increasing the size (over the range tested) of the additional stimuli was not effective in producing further reductions in $\mathrm{AK}$ when the increase in size merely served to place additional contour further into the periphery of the visual field. However, when, as in Experiment 2, the increased additional contour is confined to the central visual field, in the form of an annulus surrounding the AKS, greater reductions in AK result. Additional contour in the near periphery appears to enhance fixational stability in all directions, thus reducing $\mathrm{AK}$ in all dimensions of the visual field.

Our results also indicate that peripherally placed stimuli can influence the reported direction of AK. In Experiment 1 , additional stimuli oriented vertically produced significantly more vertical AK than did those oriented horizontally; however, horizontally oriented stimuli did not produce more horizontal AK. The two stimulus configurations differed, however (see Results and Discussion for Experiment 1); therefore, it remains a possibility that 
a stimulus oriented in one direction selectively enhances fixational stability in the opposite dimension of the visual field.

A second indication of the directional influence of peripheral stimuli is that those placed $1^{\circ}$ to one side of the AKS in Experiment 1 increased the amount of AK in the opposite direction. This apparently confirms the finding of Uchiyama and Tsuji (1963). In Experiment 2, however, the placement of stimuli $5^{\circ}$ eccentric to the AKS in four orientations did not systematically influence direction of AK. The effect may be limited to peripheral stimuli at very near eccentricities (about $1^{\circ}$ ).

The results of both experiments are consistent with the two prevailing theories of autokinesis, the small eyemovement theory (Matin \& MacKinnon, 1964) and the outflow or efferent theory (Leibowitz et al., 1983; Post \& Leibowitz, 1985; Whiteside, Graybiel, \& Niven, 1965). Since both rely on fixational instability as the cause of AK, both make similar predictions concerning the influence of stimuli that may serve to increase or decrease fixational stability.

\section{REFERENCES}

EDWARDs, W. (1954). Autokinetic movement of very large stimuli. Journal of Experimental Psychology, 48, 493-495.

Graybiel, A., \& Clark, B. (1945). The autokinetic illusion and its significance in night flying. Journal of Aviation Medicine, 16, 111-115.
Karwoski, T., Redner, H., \& Wood, W. (1948). Autokinetic movement of large stimuli. Journal of General Psychology, 39, 29-37.

LEVY, J. (1972). Autokinetic illusion: A systematic review of theories, measures, and independent variables. Psychological Bulletin, 78, 457-474.

Leibowitz, H. W., Shupert, C. L., Post, R. B., \& Dichgans, J. (1983). Autokinetic drifts and gaze deviation. Perception \& Psychophysics, 33, 455-459.

Matin, L., MacKinnon, G. (1964). Autokinetic movement: Selective manipulation of directional components by image stabilization Science, 143, 147-148

Post, R. B., \& Leibowitz, H. W. (1985). A revised analysis of the role of efference in motion perception. Perception, 14, 631-643.

Post, R. B., Letrowitz, H. W., \& Shupert, C. L. (1982). Autokinesis and peripheral stimuli: Implications for fixational stability. Perception, 11, 477-482.

Royce, H., Carran, A., Aftanas, M., lehman, R., \& BlumenTHAL, H. (1966). The autokinetic phenomenon: A critical review. Psychological Bulletin, 65, 243-260.

Royce, H., Stayton, W., \& Kinkade, R. (1962). Experimental reduction of autokinetic movement. American Journal of Psychology, 75 221-231.

Sadler, T., MefFerd, P., \& Weiland, B. (1966). Extent, direction and latency of autokinetic movement as a function of placement of an adjacent light. Perceptual \& Motor Skills, 23, 1087-1096.

UchiYama, M., \& Tsusi, K. (1963). The field force as measured by the apparent movement of a small light point. Japanese Psychological Research, 5, 63-71.

Whiteside, T., Graybiel, A., \& Niven, J. (1965). Visual illusions of movement. Brain: A Journal of Neurology, 88, 193-210.

(Manuscript received August 27, 1986; revision accepted for publication October 5, 1987.) 